\title{
More effective theory of nuclear forces
}

\section{Michael C. Birse*}

School of Physics and Astronomy, The University of Manchester, Manchester, M13 9PL, U.K.

E-mail: mike.birse@manchester.ac.uk

I outline why the renormalisation group is needed to analyse the scale dependence and hence determine the power counting for effective theories of strongly interacting systems. I summarise the results of several such analyses for two- and three-body forces in nuclear physics. These show that a number of terms should be significantly promoted relative to naive dimensional analysis.

6th International Workshop on Chiral Dynamics

July 6-10 2009

Bern, Switzerland

\footnotetext{
* Speaker.
} 


\section{The problem with building an EFT for nuclear forces}

Chiral perturbation theory [1] is an effective field theory (EFT) that can provide a systematic expansion of hadronic observables in powers of ratios of low-energy scales $Q$ (momenta, $m_{\pi}, \ldots$ ) to scales of the underlying physics $\Lambda_{0}\left(m_{\rho}, M_{N}, 4 \pi F_{\pi}, \ldots\right)$. Its terms are organised by naive dimensional analysis, or "Weinberg power counting", which simply counts powers of the lowenergy scales. This theory is perturbative, as its name implies, working for weakly interacting systems such as low-energy pions, photons and up to one nucleon. In contrast, nucleons interact strongly at low-energies, forming bound states (nuclei). To describe them, some interactions must be treated nonperturbatively.

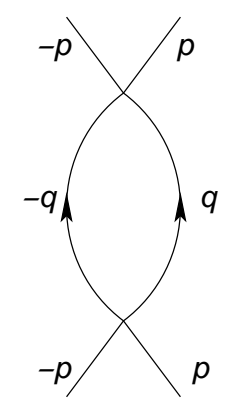

Figure 1: Loop diagram for two-body scattering

To see why this might lead to a problem with extending the theory to two or more nucleons, look at the nonrelativistic loop diagram for NN scattering in Fig. 1. For contact interactions, this has the form

$$
\frac{M}{(2 \pi)^{3}} \int \frac{\mathrm{d}^{3} q}{p^{2}-q^{2}+\mathrm{i} \varepsilon}=-\mathrm{i} \frac{M p}{4 \pi}+\text { analytic in } p^{2} .
$$

It is enhanced to order $Q$, instead of $Q^{2}$ as in the relativistic case. Nonetheless, the leading terms of the potential are of order $Q^{0}$ (OPE and the simplest contact interaction) [2] and so each iteration is suppressed by a power of $Q / \Lambda_{0}$. The theory is therefore still perturbative, provided $Q<\Lambda_{0}$.

In fact the analytic part of the integral (1.1) is linearly divergent and so we need to either cut it off or subtract it at some scale $q=\Lambda$. Iterating the potential then leads to contributions with powers of $\Lambda / \Lambda_{0}$. These will again be perturbative provided $\Lambda<\Lambda_{0}$, and so cannot generate bound states.

The commonly used workaround for this is the "Weinberg prescription", where people expand the potential to some order in $Q$ and then iterate it to all orders in their favourite dynamical equation (Schrödinger, Lippmann-Schwinger, ... ) [2]. This has been widely applied (and even more widely invoked) in nuclear physics, but it leads to results for observables with no clear power counting. In particular, it resums a subset of terms to all orders in $Q$, some of which depend on the regulator but are of higher order than any of the terms kept in the potential. There are thus no counterterms to renormalise the results and cancel this regulator dependence. This need not be a problem provided these higher-order terms are small. But it is if we want to use these terms to generate bound states.

This issue has led to vigorous debate over the last twelve or more years and has left the nuclear EFT community polarised around two broad philosophies. One, the orthodox view, can be caricatured as "The Prophet of EFT gave us the Power Counting in the holy texts, Phys Lett B251 
and Nucl Phys B363." The other, liberal approach can be summarised as "Let the renormalisation group decide!" In terms of citations by the wider community, I must admit that the orthodox party seems to be winning the election, at least so far. In this contribution, I shall try to summarise the liberal point of view.

\section{Renormalisation group}

The renormalisation group (RG) is a general tool for analysing scale dependence of systems in quantum or statistical mechanics. This means it can be used to determine the power counting that organises the effective theory describing a system. The RG was therefore an important piece of the motivation for the first chiral EFT, as noted by the Prophet himself [3].

The basic steps involved are as follows. First, we should identify all the relevant low-energy scales, $Q$. Of particular interest are any that promote leading-order terms to order $Q^{-1}$ since these can, and must, be iterated to all orders. (Iterations of these are not suppressed since each contributes a factor of order $Q^{-1}$ which cancels the $Q$ from the loop integration.) Examples of such scales in NN scattering are ones for the S-wave scattering lengths, $1 / a \lesssim 40 \mathrm{MeV}[4,5]$. Then there is the "unnatural" strength of OPE, which is set by

$$
\lambda_{N N}=\frac{16 \pi F_{\pi}^{2}}{g_{A}^{2} M_{N}} \simeq 290 \mathrm{MeV} .
$$

This is built out of high-energy scales $\left(4 \pi F_{\pi}, M_{N}\right)$ but its magnitude is only $\sim 2 m_{\pi}$, suggesting that it may be better treated as a low-energy scale.

Next, we cut off the theory at some arbitary scale $\Lambda$, above the low-energy scales $Q$ but below the scale $\Lambda_{0}$ of the underlying physics, as in Fig. 2. (This assumes good separation of these scales, as required for a convergent expansion of the resulting EFT.)

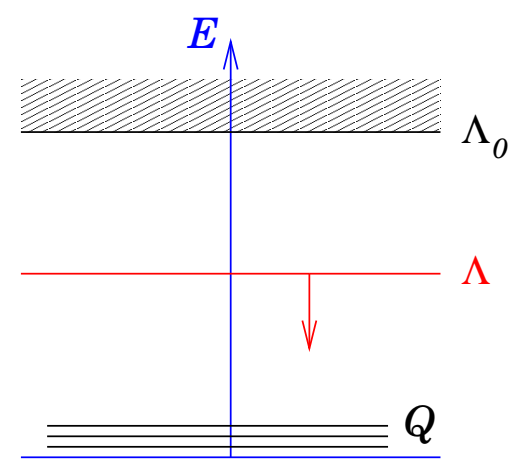

Figure 2: The running cutoff $\Lambda$.

Then we can follow the evolution of our theory as we "integrate out" more and more of the physics by lowering $\Lambda$. (Don't even think about taking $\Lambda$ to infinity here!) As we vary this arbitrary cutoff, we demand that physics (for example, the $T$ matrix) be independent of $\Lambda$.

Finally we rescale the theory by expressing all dimensioned quantities in units of $\Lambda$ so that we can look for fixed points of the RG flow-the $\Lambda$-independent end points that describe scalefree systems. We can expand our EFT around one of these using perturbations that scale with 
definite powers of $\Lambda$. This scaling corresponds directly to the order in the usual power counting, $\Lambda^{v}$ corresponding to a term in the EFT of order $Q^{d}$ where $d=v-1$. If you like, we have used $\Lambda$ to stand in for all our low-energy scales since it acts as the largest acceptable low-energy scale. ${ }^{1}$

\section{Fixed points of short-range forces}

Let me illustrate these ideas with the simplest example relevant to nuclear physics: two particles interacting via short-range forces only (the pionless EFT). In this case we find two fixed points of the RG flow [8]. One of them is the trivial fixed point $V_{0}=0$. Potentials near this point describe weakly interacting systems. Their expansion around the fixed point can be organised according to the usual perturbative ("Weinberg") power counting.

Of more interest is the nontrivial fixed point. For a sharp momentum cutoff this is

$$
V_{0}(p, \Lambda)=-\frac{2 \pi^{2}}{M \Lambda}\left[1-\frac{p}{2 \Lambda} \ln \frac{\Lambda+p}{\Lambda-p}\right]^{-1}
$$

where $p=\sqrt{M E}$ is the on-shell momentum. This potential is of order $Q^{-1}$ and so it must be iterated. It describes scattering in the "unitary limit", where the scattering length $a \rightarrow \infty$. The expansion around this point has the form

$$
V(p, \Lambda)=V_{0}(p, \Lambda)+V_{0}(p, \Lambda)^{2} \frac{M}{4 \pi}\left(-\frac{1}{a}+\frac{1}{2} r_{e} p^{2}+\cdots\right) .
$$

The factor $V_{0}^{2} \propto \Lambda^{-2}$ multiplying each of the perturbations promotes them by two orders compared to naive expectations. This leads to the "KSW" power counting for systems with large scattering lengths $[4,5]$. The terms in the resulting expansion correspond directly to the terms in the effectiverange expansion. ${ }^{2}$

Two-body scattering is sufficiently simple that we do not really need the full power of the RG to understand the origin of this enhancement; it follows from the form of the wave functions at short distances. Two particles in the unitary limit are described by irregular solutions of the Schrödinger equation. At small radii (in $S$ waves) these behave as $\psi(r) \propto r^{-1}$. Any cutoff smears a contact interaction over range $R \sim \Lambda^{-1}$. We therefore need the extra factor of $\Lambda^{-2}$ in the interaction in order to cancel the cutoff dependence from $|\psi(R)|^{2} \propto \Lambda^{2}$ in its matrix elements.

The same idea can also be used to understand the even stronger promotion of three-body forces in systems of three bosons or three distinct fermions near the unitary limit (for example the triton). Here, naive dimensional analysis would suggest that the leading contact term is of order $Q^{3}$ [2].

\footnotetext{
${ }^{1}$ The RG identifies which terms in an EFT must be iterated to all orders, and which should be treated as perturbations. Despite my comments about $\Lambda$ above, we may use the resulting effective potential with a cutoff above $\Lambda_{0}$, but only if we respect the counting the RG gives us. That is, we must iterate all terms of order $Q^{-1}$ and we must not iterate any "irrelevant" terms of order $Q^{+d}$. Otherwise we might, if we are very lucky, discover a new power counting, as in [6]. But, more usually, we lose any consistent counting, as found by the many people who have tried iterating effective-range terms in short-range potentials, as in e.g. [7].

${ }^{2}$ This illustrates a general feature of EFTs: the contact interactions are directly related to observables. In this case the connection is to the phase shifts via the effective-range expansion. When long-range forces are included, the connection is either via a DW Born expansion (for weakly interacting systems) or a DW effective-range expansion (for strong short-range interactions) [9].
} 
However, as the hyperradius $R \rightarrow 0$, the three-body wave functions have the form $\psi(R) \propto R^{-2 \pm \mathrm{is}_{0}}$, with $s_{0} \simeq 1.006$. The $R^{-2}$ divergence requires that the leading three-body force be promoted by four orders, to order $Q^{-1}$. The oscillatory behaviour associated with the imaginary part of the exponent is the origin of the Efimov effect [10]. It means that the RG flow actually tends to a limit cycle instead of a fixed point $[11,12]$.

\section{Effects of iterated one-pion exchange forces}

The central piece of OPE is the only one that contributes to scattering in spin-singlet waves. This has a $1 / r$ singularity, which is not enough to alter the power-law forms of the wave functions at small $r$. The scattering in singlet waves with $L \geq 1$ is weak, and so the corresponding effective potential can be expanded using Weinberg power counting. In contrast, the ${ }^{1} S_{0}$ channel has a lowenergy virtual state. The expansion of its potential is like the one around the unitary fixed point, and can be organised using a KSW-like power counting.

The tensor piece of OPE is important in spin-triplet waves. It has a strong, $1 / r^{3}$, singularity at the origin. The resulting short-distance wave functions have the form $\psi(r) \propto r^{-1 / 4}$, multiplied by either a sine or an exponential function of $1 / \sqrt{\lambda_{N N} r}$. As a result, short-range interactions are strongly promoted in these waves and so a new power counting needed, as observed by Nogga et al. [6]. This gives the leading contact interaction an order $Q^{-1 / 2}$ in waves with $L=1,2[13,14]$. The perturbative expansion for a potential of this order will converge very slowly and so it may be simpler to iterate it along with OPE.

Note that the importance of tensor OPE does depend on energy, and hence of the cutoff $\Lambda$. At least at low energies, the centrifugal barrier protects the higher partial waves from probing the singular core of the potential. Only waves above some critical momentum are able to resolve singularity and hence require a nonperturbative treatment of OPE. For waves with $L \geq 3$ this momentum is $p_{c} \gtrsim 2 \mathrm{GeV}$ and so Weinberg power counting can be used for the usual choices of cutoff, $\Lambda \lesssim 600 \mathrm{MeV}$. In contrast, waves with $L \leq 2$ have critical momenta $p_{c} \lesssim 3 m_{\pi}$ and so NTvK counting is needed. ${ }^{3}$

Turning now to three-body forces, the two-pion exchange interaction is purely long-range and so it is not renormalised. Its leading contribution is thus of order $Q^{3}$, as in Weinberg's counting. Other contributions, involving two- or three-nucleon contact operators, are affected by the shortdistance behaviour of the wave functions.

One-pion exchange interactions are represented by the diagram in Fig. 4. The coefficients of the two-nucleon-one-pion contact operators are generically denoted by $c_{D}$ [15]. The most important of these operators is the well-known one that couples the ${ }^{1} S_{0}$ and ${ }^{3} S_{1} \mathrm{NN}$ channels [16]. This is promoted to order $Q^{5 / 4}$ by the nonperturbative treatment of the leading two-body forces. ${ }^{4}$ In addition, there will be strong promotion of operators that couple $S$ and $P$ waves, and of those that couple various combinations of $P$ and $D$ waves.

\footnotetext{
${ }^{3}$ In the ${ }^{3} S_{1}$ case, there is a further enhancement, similar to that in the ${ }^{1} S_{0}$ wave, associated with the deuteron bound state.

${ }^{4}$ The wave function for a low-energy bound state in the ${ }^{3} S_{1}$ channel is slightly less singular near the origin than that in the ${ }^{1} S_{0}$ channel, as a result of tensor OPE [13].
} 


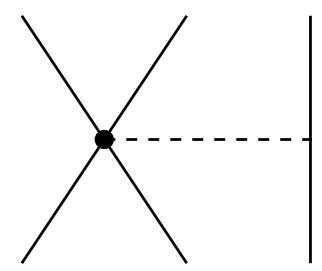

Figure 3: A three-body OPE interaction.

Finally, we have the three-body contact interaction, whose strength is denoted by $c_{E}$ [15]. The order of this term in the presence of tensor OPE is not known. I would expect it to be promoted, but by less than in pionless case. Determining the counting for this force will entail solving the three-body problem with $1 / r^{3}$ potentials. This is in progress [17].

\section{A new road map for nuclear EFT}

\begin{tabular}{l|c|c}
\hline Order & $\mathrm{NN}$ & $\mathrm{NNN}$ \\
\hline$Q^{-1}$ & ${ }^{1} S_{0},{ }^{3} S_{1} C_{0}{ }^{\prime} \mathrm{s}, \mathrm{LO} \mathrm{OPE}$ & \\
\hline$Q^{-1 / 2}$ & ${ }^{3} P_{J},{ }^{3} D_{J} C_{0}$ 's & \\
$Q^{0}$ & ${ }^{1} S_{0} C_{2}$ & \\
$Q^{1 / 2}$ & ${ }^{3} S_{1} C_{2}$ & ${ }^{1} S_{0}-{ }^{3} S_{1} C_{D 0} \mathrm{OPE}$ \\
$Q^{5 / 4}$ & & \\
$Q^{3 / 2}$ & ${ }^{3} P_{J},{ }^{3} D_{J} C_{2}$ 's & \\
$Q^{7 / 4}$ & & ${ }^{3} P_{0}-{ }^{1} S_{0} C_{D 0} \mathrm{OPE}$ \\
$Q^{2}$ & ${ }^{1} S_{0} C_{4},{ }^{1} P_{1} C_{0}, \mathrm{NLO} \mathrm{OPE}, \mathrm{LO} \mathrm{TPE}$ & ${ }^{3} P_{1}-{ }^{3} S_{1} C_{D 0} \mathrm{OPE}$ \\
$Q^{5 / 2}$ & ${ }^{3} S_{1} C_{4}$ & ${ }^{3} P_{J},{ }^{3} D_{J} C_{D 0}$ ' OPE \\
$Q^{3}$ & $\mathrm{NLO} \mathrm{TPE}$ & $\mathrm{LO} 3 \mathrm{~N} \mathrm{TPE}$ \\
$Q^{?}$ & & $C_{E}$ \\
\hline
\end{tabular}

Table 1: Orders of terms in the two- and three-nucleon effective potentials for waves with $L \leq 2$. The leading coefficient in each interaction is labelled by the subscript 0 , a subleading one (with one power of the energy or two derivatives) by the subscript 2 , and so on.

The results of the various RG analyses of nuclear forces that I have outlined above are summarised in Table 1. This shows all two- and three-body forces up to order $Q^{3}$, which would correspond to $\mathrm{N}^{2} \mathrm{LO}$ in Weinberg's power counting. As already discussed, the ones of order $Q^{-1}$ must be iterated, and it is probably most convenient to iterate also those of order $Q^{-1 / 2}$. The remainder ought to be treated as perturbations. ${ }^{5}$

\footnotetext{
${ }^{5}$ In practice, treating parts of the potential in perturbation theory does not mesh well with standard many-body methods, which treat the whole potential to all orders. Nonetheless, we can still use these methods with potentials from EFTs, provided we are careful. The unrenormalised divergences that could destroy the power counting must be kept small. This can be achieved by using a cutoff $\Lambda$ that is well below the $\Lambda_{0}$, the breakdown scale of the EFT. The price for doing this is the introduction of large artefacts $\propto(Q / \Lambda)^{n}$, so that the radius of convergence of the EFT becomes $\Lambda$ rather than $\Lambda_{0}$. Keeping these artefacts small leaves us with only a narrow window of acceptable cutoffs, just below $\Lambda_{0}$.
} 
Among the two-body interactions, the leading contact interactions in the ${ }^{3} D_{J}$ waves, the subleading ones in the $P$ and $D$ waves, and the $S$-wave $C_{4}$ 's would be absent from the corresponding $\mathrm{N}^{2} \mathrm{LO}$ potential in Weinberg counting. However all but the subleading $D$-wave interactions are present in the widely used $\mathrm{N}^{3} \mathrm{LO}$ potential.

The three-body OPE forces are more interesting. Only the $S$-wave $C_{D 0}$ term is included in the state-of-the-art $\left(\mathrm{N}^{2} \mathrm{LO}\right)$ three-body interaction. The promotion of the pion couplings for other channels could be of practical importance since $P$ waves have been implicated in several observables that cannot be described by the current three-body forces (see e.g. [18]).

\section{Acknowledgments}

I thank the INT, Seattle for its hospitality in April-June 2009, and the organisers of the program INT-09-1 "Effective field theories and the many-body problem" for forcing me to organise my thoughts on these issues.

\section{References}

[1] S. Weinberg, Physica A96 (1979) 327.

[2] S. Weinberg, Phys. Lett. $\mathbf{B 2 5 1}$ (1990) 288; Nucl. Phys. $\mathbf{B 3 6 3}$ (1991) 3.

[3] S. Weinberg, contribution to these proceedings [arXiv:0908.1964].

[4] U. van Kolck, Nucl. Phys. A645 (1999) 273 [nucl-th/9808007].

[5] D. B. Kaplan, M. J. Savage, and M. B. Wise, Nucl. Phys. B534 (1998) 329 [nucl-th/ 9802 075].

[6] A. Nogga, R. G. E. Timmermans and U. van Kolck, Phys. Rev. C72 (2005) 054006 [nucl-th/0506005].

[7] D. R. Phillips, S. R. Beane amd T. D. Cohen, Ann. Phys. 263 (1998) 255 [hep-th/9706070]

[8] M. C. Birse, J. A. McGovern and K. G. Richardson, Phys. Lett. B464 (1999) 169 [hep-ph/9807302].

[9] T. Barford and M. C. Birse, Phys. Rev. C67 (2003) 064006 [hep-ph/ 0206146$].$

[10] V. N. Efimov, Sov. J. Nucl. Phys. 12 (1971) 589.

[11] P. F. Bedaque, H.-W. Hammer and U. van Kolck, Phys. Rev. Lett. 82 (1999) 463 [nucl-th/9809025].

[12] T. Barford and M. C. Birse, J. Phys. A: Math. Gen. 38 (2005) 697 [nucl-th/ 0406008 ].

[13] M. C. Birse, Phys. Rev. C74 (2006) 014003 [nucl-th / 0507077 ].

[14] M. C. Birse, Phys. Rev. C76 (2007) 034002 [arXiv: 0706.0984 ].

[15] E. Epelbaum, A. Nogga, W. Gloeckle, H. Kamada, U.-G. Meissner and H. Witala, Eur. Phys. J. A15 (2002) 543 [nucl-th/0201064].

[16] C. Hanhart, U. van Kolck and G. A. Miller, Phys. Rev. Lett. 85 (2000) 2905 [nucl-th/ 0004033 ].

[17] L. Platter, work in progress.

[18] R. Machleidt, arXiv:0909.2881. 